\title{
Cytotoxicity of hydroxyapatite-tyrosine complex with gray titania coating on titanium alloy surface to L929 mouse fibroblasts
}

\author{
Kanda LEELANARATHIWAT ${ }^{1}$, Kentaro MINATO ${ }^{1}$, Yasuhiro KATSUTA ${ }^{2}$, Yuichi OTSUKA ${ }^{3}$, Hiroaki KATSURAGI ${ }^{4}$ \\ and Fumihiko WATANABE ${ }^{1,2}$ \\ ${ }^{1}$ Functional Occlusal Treatment, The Nippon Dental University Graduate School of Life Dentistry at Niigata, 1-8 Hamaura-cho, Chuo-ku, Niigata-shi, \\ Niigata 951-8580, Japan \\ ${ }^{2}$ Department of Crown \& Bridge Prosthodontics, The Nippon Dental University School of Life Dentistry at Niigata, 1-8 Hamaura-cho, Chuo-ku, \\ Niigata-shi, Niigata 951-8580, Japan \\ ${ }^{3}$ Department of System Safety, Nagaoka University of Technology, 1603-1 Kamitomioka, Nagaoka-shi, Niigata 940-2188, Japan \\ ${ }^{4}$ Department of Oral Microbiology, The Nippon Dental University School of Life Dentistry at Niigata, 1-8 Hamaura-cho, Chuo-ku, Niigata-shi, Niigata \\ 951-8580, Japan \\ Corresponding author, Kanda LEELANARATHIWAT; E-mail: kanda@ngt.ndu.ac.jp
}

Hydroxyapatite particles (HAp) have been widely used by many dental implant systems as an implant coating material because of their osteoconductive properties. This study aimed at improving the antibacterial effect of HAp as a substitute for antibiotic agents which can increase drug resistance. HAp/gray titania was selected as the coating material for on the titanium alloy substrate due to its antibacterial properties after photocatalytic reaction. When combined with amino acids, HAp can form a fluorescent complex which enhances this property. Before clinical application, this new coating should be examined for cytotoxic effects against biological cells or tissues. Therefore, L929 mouse fibroblasts were used to represent fibrous tissue surrounding dental implant. After performing a 6-day alamarBlue assay, the new coating method using hydroxyapatite-tyrosine complex with gray titania on titanium alloy surface can be said to have no influence on the growth of fibroblasts.

Keywords: Titanium implant surface, Hydroxyapatite, Gray titania, Fluorescent complex, Cytotoxicity

\section{INTRODUCTION}

The success of the dental implant is related to the quality of osseointegration. Direct implant-bone contact without fibrous tissue formation has been proved to provide long-term clinical success due to implant stability. Important factors that strongly affect bone anchoring are implant surface characteristics and composition ${ }^{1-3)}$. Many attempts have been made to develop new implant surface materials, which can enhance biomechanical implant stability. Recently, the trend of surface modification has shifted to focus on nanotechnology due to cell surface-substrate interactions. Titania nanotubes and various nanoparticles have been developed to use as new coating materials, however, one of the most common problems is the difficulty of fabricating a controlled uniform coating surface ${ }^{4,5)}$.

The tissue response after dental implantation is comparable to the normal healing process of bone fracture because healing quality is dependent on implant surface characteristics and primary stability. Dental implant topographic properties should be responsive to high surface energy to enhance migration, adhesion and proliferation of osteoblasts and provide strong affinity for adsorption of related extracellular substances surrounding the dental implant, such as proteins for fibrin matrix formation ${ }^{2,6,7)}$.

Color figures can be viewed in the online issue, which is available at J-STAGE.

Received Sep 7, 2018: Accepted Oct 22, 2018

doi:10.4012/dmj.2018-296 JOI JST.JSTAGE/dmj/2018-296
Hydroxyapatite particles (HAp) have been widely used by many implant systems as a coating material as they are osteoconductive. Calcium phosphate molecules can facilitate the adsorption of many biological substances to the implant surface. Fibronectin and vitronectin are proteins that are involved in the formation of fibrin matrix, which acts as a bone scaffold for osteogenesis, around the dental implant and these proteins promote faster cell differentiation and earlier primary stability ${ }^{6,7)}$. Moreover, HAp might increase the surface area and promote corrosion resistance of the dental implant ${ }^{7)}$.

Successful osseointegration of the dental implant is dependent not only on host cell attachment but also prevention of oral bacteria invasion. Once the implant is placed in the patient's mouth, the implant surface can quickly become occupied by many microorganisms, which are a barrier for osseointegration. Many studies have attempted to develop an implant surface that is capable of both inhibiting bacterial attachment and possessing bactericidal activity to prevent short- and long-term bacterial infection ${ }^{5}$. Titanium dioxide $\left(\mathrm{TiO}_{2}\right)$ or titania is an excellent photocatalyst, which is used as a semiconductor in the photocatalytic reaction because of its nontoxicity and high chemical stability. Because of its specific band gap, the effective photon wavelength that can be used with this photocatalyst tends to be near ultraviolet (UV) light ${ }^{8,9)}$. In the dental field, $\mathrm{TiO}_{2}$ has also been used as a coating material on the implant surface. In vitro, many studies showed that the bactericidal activity 
of $\mathrm{TiO}_{2}$ is mediated through the generation of free oxygen radicals on the surface of implants with photocatalyst coating. Many oral bacteria, such as Streptococcus oralis and Staphylococcus aureus, or pathogenic bacteria like Aggregatibacter actinomycetemcomitans and Fusobacterium nucleatum are affected by this reaction ${ }^{10-12)}$. Furthermore, the photocatalytic reaction via 1 -h UV light irradiation affects multispecies biofilms, including S. sanguinis, Actinomyces naeslundii and $F$. nucleatum. However, recently, the hazard of prolonged UV light exposure to living cells has been acknowledged. Thus, the use of visible light has gained interest. Dititanium trioxide $\left(\mathrm{Ti}_{2} \mathrm{O}_{3}\right)$, instead of $\mathrm{TiO}_{2}$, was used in the plasma spraying process with HAp to produce gray titania coating, which could react to visible light and provide photocatalytic activity. Previous studies confirmed the photocatalytic reaction of HAp/gray titania under red $(650 \mathrm{~nm})$, green $(532 \mathrm{~nm})$ and blue $(425 \mathrm{~nm})$ visible light ${ }^{13,14)}$.

Recent studies have demonstrated the advantages of using intrinsic fluorescent substances combined with semi-conductive photocatalysts. 8-Hydroxyquinoline and aromatic amino acids were chosen to coat the titanium alloy surface. The results showed that the presence of fluorescent substances could help reduce the number of oral pathogenic bacteria types. However, the strong nature of fluorescence also induces great cytotoxicity to human cells, thus, aromatic amino acids are preferable ${ }^{13,14)}$.

In this study, we selected HAp/gray titania as the coating material on titanium alloy substrate and investigated its osteoconductive and antibacterial effects. In addition, tyrosine, which is an aromatic amino acid, was added by cold isostatic pressure due to its intrinsic fluorescent property. This method was presumed to result in successful osseointegration with good biocompatibility, thus allowing it to be applied as the prototype dental implant surface coating material. Visible light can be applied intraorally to the osseointegrated dental implant to activate the photocatalytic coating material, then initiate the antibacterial effect which might be useful as a nonsurgical peri-implantitis treatment. Compared to other treatment modalities, such as high power energy of laser or implant surface debridement, photocatalysis will be safer for dental implant topography and compositions due to the lower risk of mechanical or chemical damage. However, in vitro cytotoxicity of this implant surface modification to hard and soft tissues surrounding the dental implant should be investigated before clinical use.

The purpose of this study was to investigate the cytotoxicity of a new titanium alloy surface modification using luminescent HAp-amino acid complex with gray titania coating on mouse fibroblasts (L929 cells).

\section{MATERIALS AND METHODS}

\section{Sample preparation}

All samples were received from Nagaoka University of Technology, Japan, and prepared as previously described $^{14)}$. Briefly, titanium alloy (Ti6Al4V) substrate was coated with $20 \%$ wt $\mathrm{Ti}_{2} \mathrm{O}_{3}$ and $80 \%$ wt HAp (HAP100, Taihei Chemical, Osaka, Japan) using the plasma spraying method before applying tyrosine (Kishida Chemical, Osaka, Japan) to create the luminescent HAp-amino acid complex surface by cold isostatic process (Model P-500, Kobe Steel, Kobe, Japan) for 20 min at $800 \mathrm{MPa}$. The coated titanium alloy was cut into pieces measuring $10 \times 10 \times 3 \mathrm{~mm}$ under water cooling. All samples were then cleaned by gamma ray sterilization. In this study, titanium alloy without coating was also used to compare cellular toxicity with the new complex coating material. Empty wells and wells containing zinc phosphate cement (GC, Tokyo, Japan) were selected as the negative and positive controls, respectively. The sample size was decided to be $8(n=8)$ per group for each experiment condition. The details of the samples used in this study is presented in Table 1.

\section{Fibroblast cell culture and cell density adjustment}

Mouse fibroblasts (L929 cell line) were used in this study and represented soft tissue cells. The fibroblasts were cultured in Dulbecco's Modified Eagle Medium — low glucose and supplemented with 10\% fetal bovine serum, penicillin-streptomycin and amphotericin B (Merck, Darmstadt, Germany) in flasks at $37^{\circ} \mathrm{C}$ and $5 \% \mathrm{CO}_{2}$. An adequate number of cells were detached from the flasks

Table 1 Number of samples tested in each experiment

\begin{tabular}{|c|c|c|c|c|c|c|c|c|c|}
\hline \multirow{2}{*}{ Samples } & \multirow{2}{*}{ Abbr. } & \multicolumn{2}{|c|}{ Day 1} & \multicolumn{2}{|c|}{ Day 2} & \multicolumn{2}{|c|}{ Day 4} & \multicolumn{2}{|c|}{ Day 6} \\
\hline & & Ala & Cell & Ala & Cell & Ala & Cell & Ala & Cell \\
\hline Blank (-ctrl) & Con & 8 & - & 8 & 8 & 8 & 8 & - & 8 \\
\hline Zinc phosphate (+ctrl) & $\mathrm{Zn}$ & 8 & - & 8 & 8 & 8 & 8 & - & 8 \\
\hline Ti alloy & $\mathrm{Ti}$ & 8 & - & 8 & 8 & 8 & 8 & - & 8 \\
\hline Ti alloy with coating & HA & 8 & - & 8 & 8 & 8 & 8 & - & 8 \\
\hline
\end{tabular}

Ala: alamarBlue Assay, Cell: Cell counting method, Ti alloy: Titanium alloy (Ti6Al4V), Ti alloy with coating: Titanium alloy substrate coating with hydroxyapatite-tyrosine complex and gray titania. 
using $0.25 \%$ trypsin-EDTA solution. The cells were then plated onto 6-well plates (Falcon ${ }^{\circledR}$, Corning, Corning, NY, USA) in a total volume of $2 \mathrm{~mL}$ medium with $2.5 \times 10^{3}$ fibroblasts added into each well. The culture medium was replaced every 2 days.

\section{AlamarBlue assay and cell viability measurement}

A cell culture insert with a $0.4-\mu \mathrm{m}$ pore size, HD, PET track-etched membrane $\left(\right.$ Falcon $\left.^{\circledR}\right)$ was put into every well, followed by the sample and $2 \mathrm{~mL}$ of medium (Fig. 1). For the negative control group, no sample was placed in the cell culture insert. All plates were incubated at $37^{\circ} \mathrm{C}$ and $5 \% \mathrm{CO}_{2}$ for 1,2 and 4 days. After the specified incubation period, culture medium from each well was separately collected for analysis of element composition. The cell culture insert and sample were then removed from the well, and $100 \mu \mathrm{L}$ of alamarBlue dye (BioRad, Hercules, CA, USA) and $2 \mathrm{~mL}$ of medium were added to each well and incubated for an additional 24 $\mathrm{h}$ under similar conditions. Fluorescence intensity was recorded using Powerscan MX (DS Pharma Biomedical, BayBioImaging, Osaka, Japan) with 570/660 nm excitation and emission wavelengths. The fluorescence intensity value was proportional to the number of living cells, representing fibroblast cell viability.

\section{Cell counting method}

The medium was discarded from the well and $0.25 \%$ Trypsin-EDTA solution was added to detach cells. Cell suspensions were then washed and transferred to 15$\mathrm{mL}$ centrifuge tubes using a transfer pipette. Cells were then centrifuged at $4^{\circ} \mathrm{C}$ and 3,000 rpm for $10 \mathrm{~min}$. After centrifugation, the supernatant was removed and $1 \mathrm{~mL}$ of medium was added and well mixed. For the cell counting method, $11 \mu \mathrm{L}$ of cell suspension was put into a disk chamber and the number of fibroblasts was automatically calculated by a cell imaging counter (CYTORECON ${ }^{\mathrm{TM}}$, GE Healthcare Japan, Tokyo, Japan).

\section{Elemental analysis of culture medium}

The trace element analysis method was carried out to determine chemical substances in the medium of each sample. The collected medium was randomized and analyzed by a total reflection X-ray fluorescence spectrometer (OURSTEX 200TX, OURSTEX, Osaka, Japan).

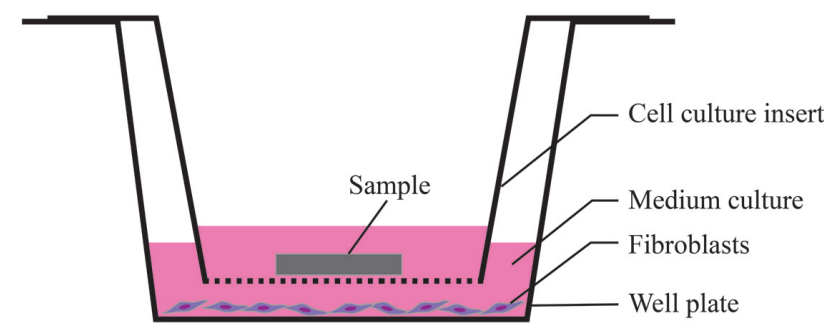

Fig. 1 Illustration demonstrating the well set up. The cell culture insert with sample is shown.

\section{Statistical analysis}

Mean and standard deviation (S.D.) of fluorescent intensity and number of cells were calculated and differences between groups were statistically analyzed by two-way analysis of variance. Regarding the interaction between two main factors, different groups and incubation time, simple main effect analysis was also conducted using the Bonferroni method. All statistical tests were performed using SPSS ${ }^{\circledR}$ Statistics 20.0 (IBM $^{\circledR}$, Armonk, NY, USA). $p$ Values $<0.05$ were considered statistically significant.

\section{RESULTS}

Cell viability measurement by alamarBlue assay

Differences in fluorescent intensities between groups were statistically analyzed by two-way ANOVA. When an interaction between two main factors was found $(p<0.05)$, then simple main effect analysis was performed. Among the tested groups, the mean fluorescent intensity values were not significantly different on days 1 and 2 .

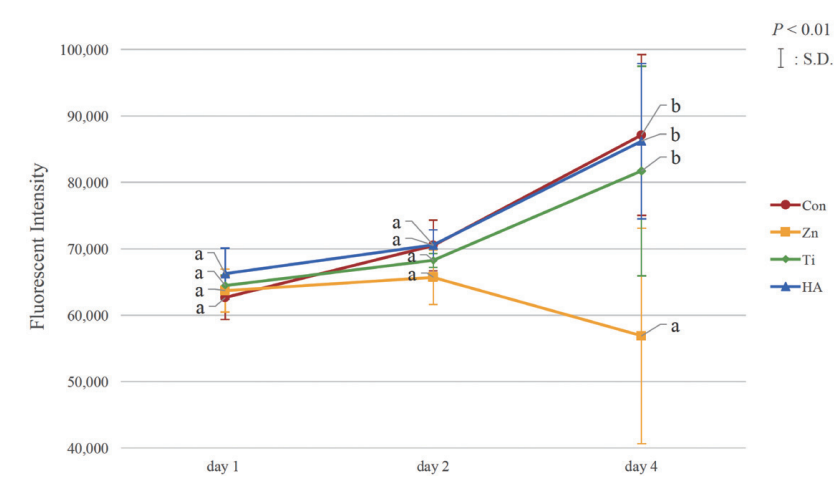

Fig. 2 Cell viability measurement by the alamarBlue assay.

Groups that do not share the same letter are significantly different $\left({ }^{*} p<0.01\right)$.

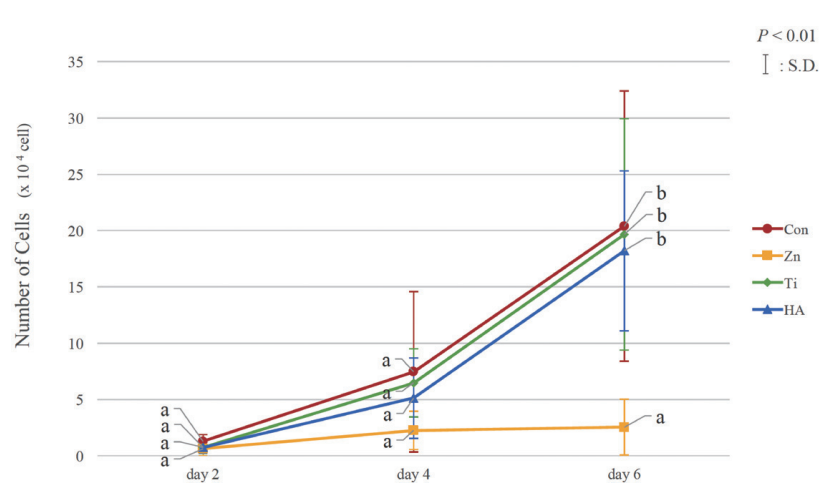

Fig. 3 Number of cells counted by the cell imaging counter.

Groups that do not share the same letter are significantly different $\left({ }^{*} p<0.01\right)$. 

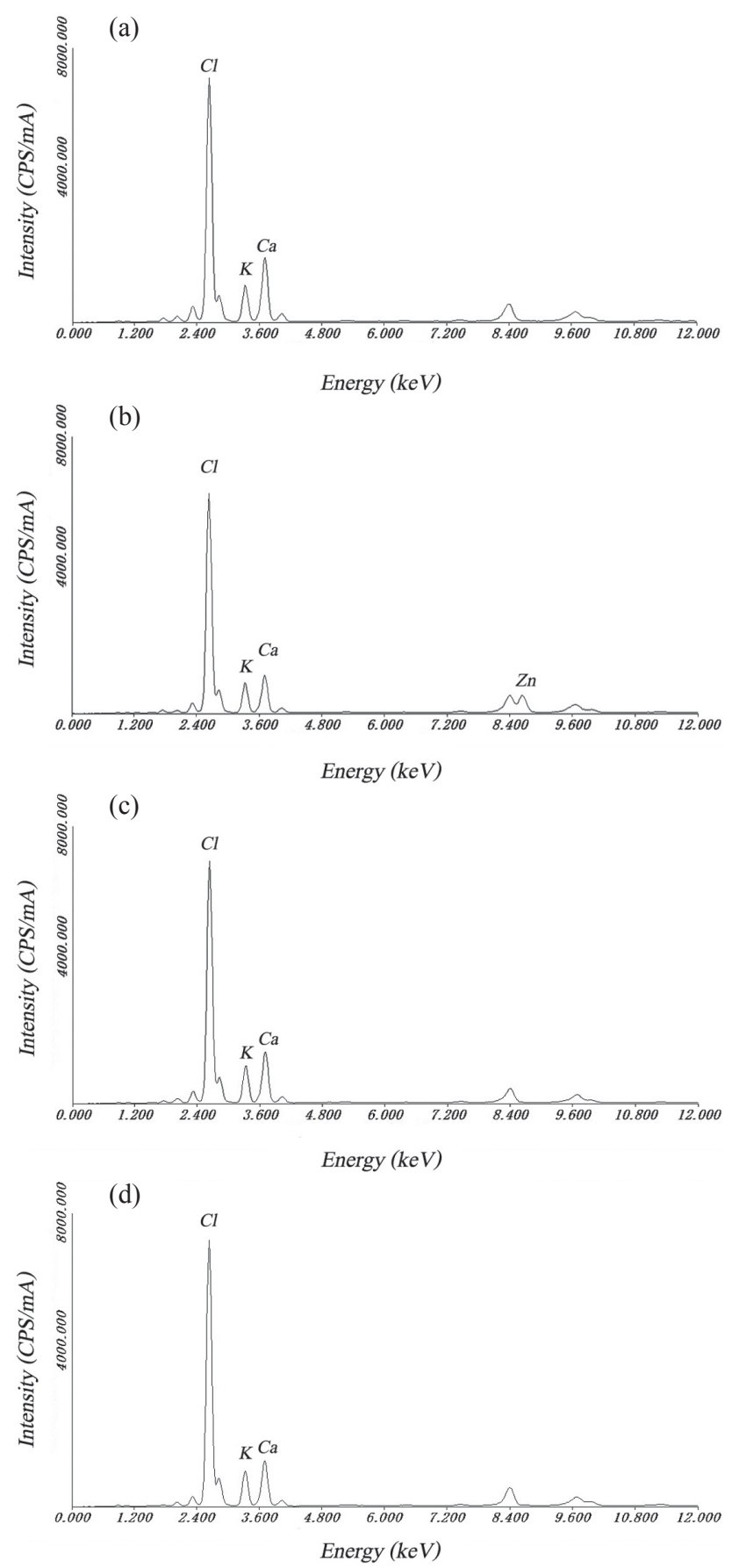

Fig. 4 Results from total reflection X-ray fluorescence spectrometry after 6 days of incubation.

(a) Culture medium only (negative control), (b) Zinc phosphate cement (positive control), (c) Titanium alloy, (d) Titanium alloy with hydroxyapatitetyrosine complex and gray titania coating.

However, the mean value of the zinc phosphate cement group (positive control) on day 4 was significantly lower than those of all other groups $(p<0.01)$. From the results for each individual day, all tested groups showed a significant increase in cell viability on day $4(p<0.01)$, except the zinc phosphate cement group (Fig. 2).

\section{Cell counting method}

Differences in cell numbers between groups were statistically analyzed by two-way ANOVA. The interaction between two main factors was also presented $(p<0.05)$. Among the test groups, the mean number of cells was not significantly different on days 2 and 4 . In contrast, cell numbers on day 6 in the zinc phosphate cement group were significantly lower than those of the other groups $(p<0.01)$. In addition, all tested groups exhibited a significant increase in cell numbers on day $6(p<0.01)$, excluding the zinc phosphate cement group (Fig. 3).

\section{Elemental analysis}

The elemental analysis of collected medium was performed by total reflection X-ray fluorescence spectrometry. All tested groups showed similar components in their medium (Figs. 4a-d). Chloride (Cl) and calcium $(\mathrm{Ca})$ were found and considered as nutrition in the medium, and potassium $(\mathrm{K})$ was revealed as the cell component substance. A difference in the culture medium composition was found in the zinc phosphate cement group (Fig. 4d), was the only group that contained zinc ion.

\section{DISCUSSION}

The importance of implant surface modification has been mentioned by several studies ${ }^{2,6,7)}$. Implant surface energy might play a significant role in adsorption of extracellular fibrin matrix, which forms after implantation. This protein fibrin matrix is a bone scaffold for osteogenic cells. For this reason, implant surface technology has been continually developed.

Our new coating material was composed of three components: $\mathrm{Ti}_{2} \mathrm{O}_{3}$, Hydroxyapatite and tyrosine amino acid. The first two components were applied by plasma spraying method on the titanium alloy substrate and the last one was added by CIP pressure. The previous study showed that the plasma-sprayed process can transform $\mathrm{Ti}_{2} \mathrm{O}_{3}$ into $\mathrm{TiO}_{2}$ (Rutile) with mixed phase due to heating. However, the coating surface still presented as darkgray color because $\mathrm{Ti}^{3+}$ was remained in the interstitial sites in the lattice of rutile $\mathrm{TiO}_{2}{ }^{14)}$.

In this study, we can prove the absence of the effect of hydroxyapatite-tyrosine complex with gray titania coating on the growth of L929 mouse fibroblasts after exposuring the coating material surface to the culture medium for 6 days. The result was conformed to another study which demonstrated no effect to the growth of mouse osteoblasts (MC3T3-E1 cells) when using the same type and method of coating materials ${ }^{15}$. It might be implied that these coating materials have a good compatibility with both hard and soft tissue cells surrounding osseointegrated dental implant. Another study about the luminescent complex with gray titania was also conducted ${ }^{14}$. Instead of tyrosine amino acid, 
they selected 8-hydroxyquinolone (8-Hq) which can also create a fluorescent complex with hydroxyapatite under visible light. Unfortunately, this material had a cytotoxic effect on osteoblastic cells.

HAp is a mineral substance, derived from calcium apatite, that has many advantages as a coating material such as osteoconductivity, increased implant surface area and corrosion resistance. Thus, HAp might enhance osseointegration and biomechanical properties of the dental implant ${ }^{6,7)}$. Therefore, HAp coating can be a desirable surface for the dental implant. The most common method of coating HAp to the implant surface is the plasma spraying method.

Tyrosine is an aromatic amino acid that can be found in human blood plasma. Its intrinsic florescent property makes tyrosine an ideal molecule for many applications ${ }^{16}$. It was expected that the combination of HAp and tyrosine might have strong biocompatibility and low cytotoxicity. Therefore, a previous study combined HAp with three aromatic amino acids ${ }^{15}$. The authors concluded that the new complex coating on titanium alloy surface did not affect the growth of mouse osteoblasts. Moreover, irradiation of this new complex coating material with visible light resulted in an antibacterial effect against Escherichia coli compared with non-complex coating titanium alloy.

However, some drawbacks have been found after application. Porosity, residual stress and HAp in various phases might be present on the modified implant surface, which could lead to delamination of the coating materials, especially when placed in a dense bone type ${ }^{1)}$. After fabricating this new complex coating material, the properties of the coating surface should be evaluated before clinical application. One of the most important issues is the quality of the coating method, the controlled homogeneity surface and reproducibility of the results, especially in the actual form of the dental implant, which consists of several small threads.

We selected the murine L929 cell line to represent the soft tissue surrounding the dental implant. Compared with human gingival fibroblasts, L929 cells have different cell structures due to their heteroploid chromosome pattern. Additionally, these two fibroblastic cell types exhibit different responses to external substances. In particular, L929 cells line display greater sensitivity to cytotoxic substances than human gingival fibroblasts. Differences in cell membrane permeability, mitochondrial function and culture medium concentration could affect the tolerance to external toxicity and cell survival ${ }^{17-19)}$. Thus, from our study results, it might be implied that the coating materials themselves would have no significant effect on the growth of human gingival fibroblasts.

Previous reports have demonstrated that zinc release can enhance cytotoxicity to L929 cells, which was related to elemental analysis results in the present study ${ }^{19-21)}$. Moreover, the $\mathrm{pH}$ of the culture medium is also related to cell viability. Koosha et al. reported that the $\mathrm{pH}$ of zinc phosphate cement medium decreased after 2 days to $6.6^{21)}$, and this finding was supported by our results. In this study, although the culture medium had buffering capacity, the $\mathrm{pH}$ of the medium from the zinc phosphate cement group dropped from 7.8 to about 6.9 after 4 days. Therefore, it should be remarked that not only zinc ion release but also the acidic condition caused by the cement could have a detrimental effect on fibroblast growth and differentiation.

Preliminary cytotoxicity testing of the coating materials on soft and hard tissue cells surrounding the dental implant showed that the materials themselves do not affect cell viability. However, the design of this study was to use the cell culture inserted to hold the tested sample in the culture medium, which is not a direct testing method. Because our aim was to inspect the effect from elution of these chemical substances that were chosen as the coating materials on the viability of the cells. Therefore, the condition of this study was decided to avoid the physical interruption caused by the titanium alloy sample. As mentioned above, this coating material was a complex of photocatalyst, therefore it should be activated by the visible light to generate the reactive oxygen species which will result in antibacterial property. Thus, the growth of the cells after light irradiation must be investigated.

The purpose of using an intrinsic fluorescent substance was to enhance the photocatalytic reaction between visible light and HAp-tyrosine complex with gray titania, which have been produced as new coating materials. A previous study on these coating materials confirmed a bactericidal effect against E.coli only, and the irradiation time was too long to perform intraorally due to the limited power of the light sources ${ }^{15}$. Future studies will investigate antibacterial properties of HAp-amino acid complex with gray titania irradiated with visible light against oral pathogenic bacteria, especially periimplantitis related bacteria. Moreover, implant surface characteristics, such as surface roughness, wettability and surface composition after coating, are crucial factors involved in dental implant osseointegration, which require additional investigation.

\section{CONCLUSION}

Within the limitations of the present study, we found the new coating material using HAp-tyrosine complex with gray titania coating on titanium alloy surface had no influence on fibroblast growth. Further studies are required to confirm the antibacterial effects of coating materials from the photocatalytic reaction against oral pathogenic bacteria involved in peri-implant inflammation.

\section{CONFLICTS OF INTEREST}

This research did not receive any specific grant from funding agencies in the public, commercial, or not-forprofit sectors. 


\section{REFERENCES}

1) Le Guehennec L, Soueidan A, Layrolle P, Amouriq Y. Surface treatments of titanium dental implants for rapid osseointegration. Dent Mater 2007; 23: 844-854.

2) Mavrogenis AF, Dimitriou R, Parvizi J, Babis GC. Biology of implant osseointegration. J Musculoskelet Neuronal Interact 2009; 9: 61-71.

3) Shibata Y, Tanimoto Y. A review of improved fixation methods for dental implants. Part I: Surface optimization for rapid osseointegration. J Prosthodont Res 2015; 59: 20-33.

4) Parnia F, Yazdani J, Javaherzadeh V, Maleki Dizaj S. Overview of nanoparticle coating of dental implants for enhanced osseointegration and antimicrobial purposes. J Pharm Pharm Sci 2017; 20: 148-160.

5) Raphel J, Holodniy M, Goodman SB, Heilshorn SC. Multifunctional coatings to simultaneously promote osseointegration and prevent infection of orthopaedic implants. Biomaterials 2016; 84: 301-314.

6) Ma T, Ge X, Zhang Y, Lin Y. Effect of Titanium Surface Modifications of Dental Implants on Rapid Osseointegration. In: Sasaki K, Suzuki O, Takahashi N, editors. Interface Oral Health Science 2016. Singapore: Springer, Singapore; 2017. p. 247-256.

7) Parithimarkalaignan S, Padmanabhan TV. Osseointegration: an update. J Indian Prosthodont Soc 2013; 13: 2-6.

8) Hashimoto K, Irie H, Fujishima $\mathrm{A} \mathrm{TiO}_{2}$ Photocatalysis: A historical overview and future prospects. Jpn J Appl Phys 2005; 44: 8269-8285.

9) Anpo M. Photocatalysis on titanium oxide catalysts: Approaches in achieving highly efficient reactions and realizing the use of visible light. Catalysis Surveys from Asia 1997; 1: 169-179.

10) Shiraishi K, Koseki H, Tsurumoto T, Baba K, Naito M, Nakayama K, et al. Antibacterial metal implant with a TiO2-conferred photocatalytic bactericidal effect against Staphylococcus aureus. Surf. Interface Anal 2009; 41: 17-22.

11) Suketa N, Sawase T, Kitaura H, Naito M, Baba K, Nakayama $\mathrm{K}$, et al. An antibacterial surface on dental implants, based on the photocatalytic bactericidal effect. Clin Implant Dent
Relat Res 2005; 7: 105-111.

12) Westas E, Hayashi M, Cecchinato F, Wennerberg A, Andersson $\mathrm{M}$, Jimbo R, et al. Bactericidal effect of photocatalyticallyactive nanostructured $\mathrm{TiO}_{2}$ surfaces on biofilms of the early oral colonizer, Streptococcus oralis. J Biomed Mater Res A 2017; 105: 2321-2328.

13) Matsuya T, Otsuka Y, Tagaya M, Motozuka S, Ohnuma K, Mutoh Y. Formation of stacked luminescent complex of 8-hydroxyquinoline molecules on hydroxyapatite coating by using cold isostatic pressing. Mater Sci Eng C Mater Biol Appl 2016; 58: 127-132.

14) Matsuya T, Morakul S, Otsuka Y, Ohnuma K, Tagaya $\mathrm{M}$, Motozuka S, et al. Visible light-induced antibacterial effects of the luminescent complex of hydroxyapatite and 8-hydroxyquinoline with gray titania coating. Appl Surf Sci 2018; 448: 529-538.

15) Morakul S, Otsuka Y, Ohnuma K, TAGAYA M, Motozuka $\mathrm{S}$, Miyashita Y, et al. Antibacterial evaluation of fluorescent HAp complex with photocatalyst coating by visible light irradiation. Paper presented at: Asia-Pacific Conference on Fracture and Strength (APCFS) 2016, 2016.

16) Teale FW, Weber G. Ultraviolet fluorescence of the aromatic amino acids. Biochem J 1957; 65: 476-482.

17) Hermann JS, Buser D, Schenk RK, Higginbottom FL, Cochran DL. Biologic width around titanium implants. A physiologically formed and stable dimension over time. Clin Oral Implants Res 2000; 11: 1-11.

18) Kwon JS, Illeperuma RP, Kim J, Kim KM, Kim KN. Cytotoxicity evaluation of zinc oxide-eugenol and non-eugenol cements using different fibroblast cell lines. Acta Odontol Scand 2014; 72: 64-70

19) Schweikl H, Schmalz G. Toxicity parameters for cytotoxicity testing of dental materials in two different mammalian cell lines. Eur J Oral Sci 1996; 104: 292-299.

20) Attar N, Tam LE, McComb D. Mechanical and physical properties of contemporary dental luting agents. J Prosthet Dent 2003; 89: 127-134.

21) Koosha S, Nematolahi F, Torshabi M, Tabatabaei F. In vitro cytotoxicity of two categories of dental cements. J Res Dentomaxillofac Sci 2016; 1: 31-35. 